\title{
Probabilistic Cooperative Coded Forwarding for Broadcast Transmissions in Industrial Mobile Edge Communications
}

\section{Wenjun Huang ( $D$ 16111027@bjtu.edu.cn )}

Beijing Jiaotong University School of Electronic and Information Engineering https://orcid.org/00000002-2765-3381

\section{Xu Li}

Beijing Jiaotong University School of Electronic and Information Engineering Yanan Liang

Beijing Jiaotong University School of Electronic and Information Engineering

\section{Research Article}

Keywords: Network coding, Mobile edge computing, Wireless communication, Probabilistic Forwarding

Posted Date: January 6th, 2022

DOI: https://doi.org/10.21203/rs.3.rs-1204482/v1

License: (c) (1) This work is licensed under a Creative Commons Attribution 4.0 International License. Read Full License 


\title{
RESEARCH
}

\section{Probabilistic cooperative coded forwarding for broadcast transmissions in industrial mobile edge communications}

\author{
Wenjun Huang ${ }^{*}, \mathrm{Xu}$ Li and Yanan Liang
}

\author{
${ }^{*}$ Correspondence: \\ 16111027@bjtu.edu.cn \\ School of Electronic and \\ Information Engineering, Beijing \\ Jiaotong University, Beijing, P. R. \\ China \\ Full list of author information is \\ available at the end of the article
}

\begin{abstract}
Mobile edge computing (MEC) has been considered as a key enabler for the industrial internet of things (IloT) to cope with the ever-increasing communication and computing demands of nodes. In consideration of the limited power and resource of the IloT nodes, it is necessary to design cost-effective data sharing mechanisms for MEC-enabled wireless industrial communication networks. In this article, we propose the probabilistic cooperative coded forwarding (PCCF) scheme based on network coding (NC) to minimize the required transmission number in both the data source and relay nodes. The data packets are encoded sparsely in a systematic coding framework so that the decoding process at the receivers can be more efficient. The relationship between the forwarding and coding parameters of the proposed scheme and the successful decoding probability are comprehensively analyzed and the approximations are numerically verified. Throughout the analysis, we find the optimal sparsity of network coding vectors and also the existence of minimum transmission numbers.
\end{abstract}

Keywords: Network coding; Mobile edge computing; Wireless communication; Probabilistic Forwarding

\section{Introduction}

With the rapid development of fifth-generation (5G) mobile communication technologies, the industrial internet of things (IIoT) [1] become increasingly intelligent and flexible in recent years. One of the key enabling technologies of IIoT is mobile edge computing (MEC), which integrates the cloud computing capabilities on the mobile IIoT node devices in the edge networks $[2,3]$. As a result, the ever-growing data can be efficiently processed in real-time or near real-time among the neighboring IIoT nodes in a self-organizing manner, and the substantial stress on the wireless links between the centralized cloud network and the edge network can be offloaded. Meanwhile, the increasing data sharing demands within the edge networks also put forward requirements to high efficient wireless networking and broadcasting techniques.

The main challenges of mobile edge networks include the constraints of battery capacity and computational resource of the IIoT nodes, and the dynamic and intermittent wireless links. The limited communication and computation capabilities mean that the design of distributed data sharing and processing mechanisms should be sufficiently simple and efficient with respect to time and energy. Besides, the 
sending of feedback for re-transmissions due to packet loss among IIoT nodes may be inefficient for data collection missions with a fixed deadline.

As a promising technology for efficient wireless communication, network coding (NC) has been widely investigated in the literature. It was first proposed for distributed mobile ad hoc networks (MANET) $[4,5,6]$ as it can improve the wireless multi-cast and broadcast performance by coded forwarding. In recent years, the application scenarios of network coding have been extended to MEC [7, 8] and device-to-device(D2D) communication $[9,10]$ in $5 \mathrm{G}$ networks. The performance improvement of network coding is two-fold. On the one hand, the data packets are encoded into coded packets at the source node using network coding schemes such as random linear network coding (RLNC) [11] . The receiver nodes which receive a sufficient number of coded packets from the source can decode the data packets without sending feedback. This helps the nodes to achieve more reliable transmissions. On the other hand, the relay nodes can also encode and forward the packets to the next-hop receivers rather than directly forwarding them $[4,8]$. As a result, the relay-coded packets help the receivers to recover the lost packets in the wireless channel, and the number of transmissions at the relays can be reduced. The advantages of network coding meet the requirement of efficient data sharing in mobile edge networks under intermittent wireless links.

In this article, we focus on making full use of the benefits of network coding in MEC-enabled IIoT scenarios. To this end, we propose the probabilistic cooperative coded forwarding (PCCF) scheme by jointly considering the coding and forwarding mechanisms. We first design the system architecture of the proposed scheme, including the encoding procedures at the source and the forwarding process at the multi-hop relay IIoT nodes. The source broadcast network-coded packets to its onehop neighbor nodes, and the relay nodes cooperatively re-encode and re-broadcast the packets. The impacts of direct and coded forwarding probability on the efficiency are then analyzed based on the percolation theory. To improve the efficiency of the network coding mechanisms and minimize the transmission number of coded packets, we adopt a systematic network coding framework and optimize the sparsity of coding vectors of coded packets.

The rest of this paper is organized as follows. In Sect. 2, the related works of network coding and broadcasting schemes are introduced. The design of the proposed scheme is presented in Sect. 3. The numerical analysis results of the proposed scheme are provided in Sect. 4. The simulation analysis results of the proposed scheme are provided in Sect.5. Finally, the conclusion is drawn in Sect. 6.

\section{Related works}

The data dissemination is one of the fundamental functions of mobile edge networks, which can be considered as a broadcasting problem. The most basic broadcasting mechanism is flooding, in which the network nodes will always directly forward the newly received packets to the others [6]. Flooding mechanism is quite simple as the nodes don't need to perform any computation at all, while it can quickly exhaust the channel resource and the received packets at destination nodes will be redundant. To alleviate the cost of flooding, some transmissions need to be pruned. One feasible way is probabilistic forwarding [12], where the nodes will only forward the newly 
received packet with a predetermined probability. As the broadcast transmissions of nodes are pruned without distinction, some necessary transmission may also be eliminated. Another method is deterministic forwarding [4], where the nodes will select a few neighbors for rebroadcasting according to their knowledge of network topology. To optimize the efficiency of deterministic forwarding, the nodes should perform sufficient maintenance on the topology information they have, which consumes a lot of channel resources. Besides, the packet erasure effect due to channel outage will also affect packet transmissions. To improve the resilience to failures and minimize the cost, the forwarding mechanisms should be more reliable and resource-efficient.

Network coding allows the source and intermediate nodes to encode the packets before transmission. This encoding method brings the connection between different data packets, which enables the receiver nodes to start decoding after receiving sufficient coded packets without sending feedback for re-transmission. To this end, the source and relay nodes should dynamically determine the number of coded packets and also their forwarding strategy [11] to ensure that the receiver nodes have a sufficient number of coded packets. The most commonly discussed NC scheme is random linear network coding (RLNC), in which the encoded packets are produced as random linear combinations of the original data packets, and the coding coefficients are selected from a predetermined finite field. The decoding performance of RLNC is related to the rank of the receiving coding matrix [13], which represents the linear irrelevance among the received coded packets. In order to keep the generated RLNC packets linear irrelevant, the size of the finite field of RLNC should be sufficiently large. As a result, the encoding and decoding procedure of RLNC is computationally complex. Moreover, it has also been discovered in [11] that the performance of RLNC suffers from probabilistic forwarding due to the "all-or-nothing" effect, which means a receiving node cannot decode any data packet until the rank of its receiving coding matrix reaches to a given threshold. An alternative NC scheme is an exclusive-or (XOR) based network coding, which is also known as COPE [14]. The receiver nodes will broadcast the information on whether the packets are received or not, like sending collective feedback. An intermediate node will listen and discover the "coding opportunity" and deterministically forward the coded packets to desire receivers [15]. In the XOR-based NC scheme, the packets are encoded with bitwise XOR operation, therefore it can also be considered as a variant of RLNC using the binary finite field. Though XOR-based NC is much simpler than RLNC schemes operating in large finite fields, the broadcasting of receiving status in COPE will lead to resource consumption just like the topology maintenance in deterministic forwarding.

To decrease the complexity of RLNC, the source nodes can increase the sparsity of coded packets $[16,17]$, which means that the coding coefficients are more likely to be zero. The increasing sparsity of coded packets will reduce the computational cost of both the encoding and decoding processes. The coded packets can also be generated in a systematic manner $[18,19]$, where the data packets will be first embedded into the coded packets one by one. These systematic packets can be instantaneously decoded and the recovered data packet can accelerate the decoding process of nodes. To the best of our knowledge, the rank distribution of sparse network coding has 


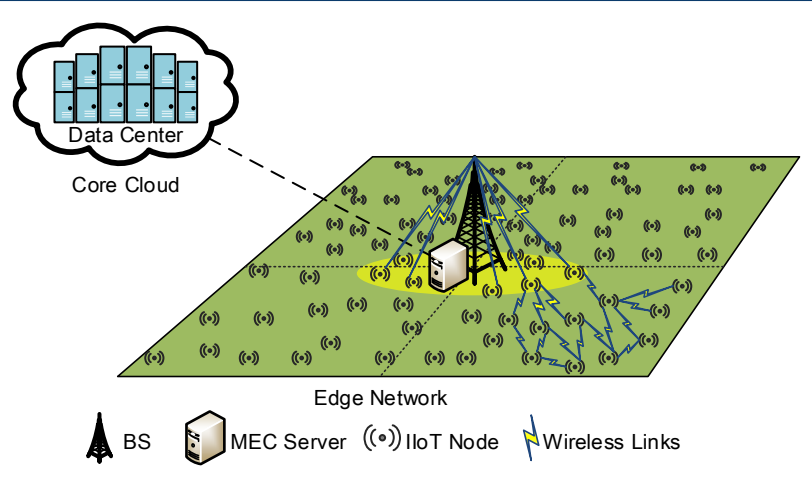

Figure 1 Architecture of MEC-enabled industrial communication network.

no closed-form expressions, which is usually provided in the approximation versions from empirical simulation results. Moreover, in a systematic network coding framework, the analysis of decoding probability should jointly consider the relationship between the received systematic and sparse coded packets.

In sum, the decoding performance at the receiver nodes is jointly affected by the forwarding probability, the number of coded packets, and the sparsity of coding vectors. The effect of these parameters should be jointly optimized so that to achieve cost-efficient data sharing.

\section{Methods}

\subsection{Network model}

We consider a MEC-enabled IIoT consisting of a base station (BS) equipped with a MEC server and $N$ randomly distributed IIoT nodes in the edge network. The MEC server has direct connections with data centers in the core cloud. The IIoT nodes in the edge network are assumed to be located inside a square area $[0, B]^{2}$ on the ground where $B$ is the edge of the square, and the BS is located at the origin of the area. We can assume that the MEC server at the BS has higher computation performance than the IIoT edge nodes. The network model is displayed in Fig. 1.

We assume a multi-hop mobile edge network scenario, where the BS can only cover a fraction of nodes. Hence the IIoT nodes which have received the packets from the BS should forward them to the rest of the nodes in the network. For the convenience of analysis, the coverage radii of the BS and the IIoT nodes are assumed to be the same and denoted as $\delta<B$, and any two nodes with distance closer than $\delta$ is assumed to have a direct link with a common packet erasure probability $\rho \in(0,1)$. This kind of network can be modeled as a Gilbert disk graph [20], one of the most studied random geometric graphs (RGG) in the literature. Let $\mathcal{N}(v)$ denote the onehop neighbor of node $v$. The cardinality of $\mathcal{N}(v)$ is referred as the degree of node $v$, i.e. $d_{v} \triangleq|\mathcal{N}(v)|$, which follows a Poisson distribution of mean $\varphi \triangleq \mathbb{E}\left(d_{v}\right)=\lambda \pi \delta^{2}$.

\subsection{Scheme Design}

The probabilistic cooperative coded forwarding scheme consists of two networkcoding based mechanisms. The BS deploys the systematic sparse network coding (SSNC) mechanism, in which it will organize $k$ data packets as a packet generation 


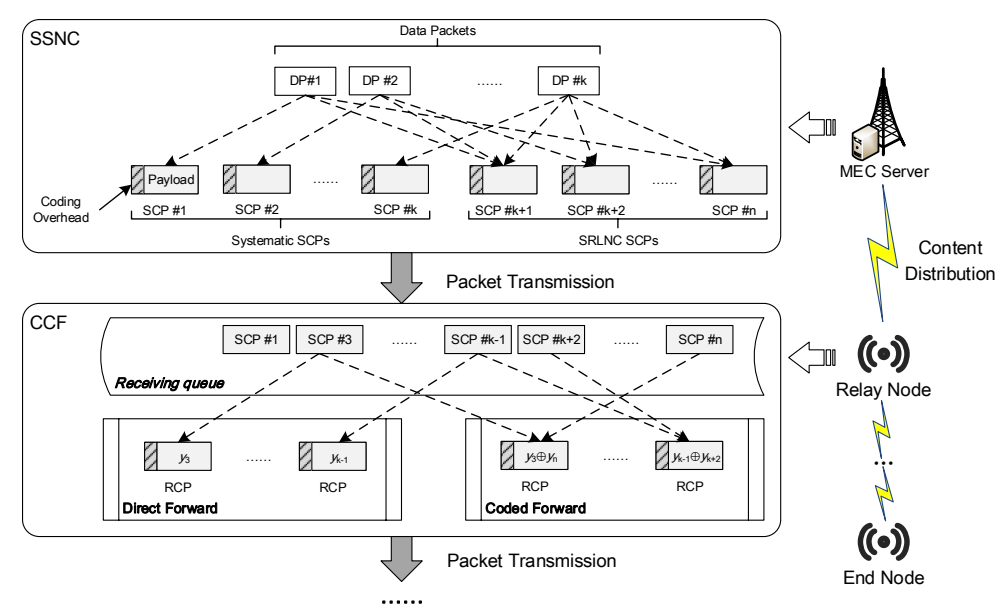

Figure 2 Diagram of the network-coding based mechanisms of PCCF.

$\mathbf{x}=\left\{x_{i} \mid i=1, \cdots, k\right\}$ and encoded them into $n \geq k$ source-coded packets (SCPs). It will broadcast the SCPs to the IIoT nodes within its coverage. An IIoT node that has received the SCPs will perform cooperative coded forwarding $(\mathrm{CCF})$, where it first directly forward the SCPs in a probabilistic manner, and then encode the received SCPs into some relay-coded packets (RCPs) and broadcast them to its onehop neighbors. In general, SSNC is a source-based network coding mechanism and $\mathrm{CCF}$ is related to the relay nodes. To accelerate the encoding and decoding process at the BS and IIoT nodes respectively, both the network coding mechanisms should be designed with low computational complexity and high efficiency. The diagram of SSNC and CCF is presented in Fig. 2, and the detailed procedures of these two mechanisms is illustrated as follows.

\subsubsection{Systematic sparse network coding (SSNC)}

In the encoding process of SSNC, the $j$-th SCP $y_{j}$ contains a coding vector $\mathbf{c}_{j}=$ $\left\{c_{j, i} \mid i=1, \cdots, k\right\}$ indicating the coefficients corresponding to the data packets, and the encoding result is given as

$$
y_{j}=\mathbf{c}_{j} \mathbf{x}=\sum_{i=1}^{k} c_{j, i} x_{i}
$$

where the operations are performed in a Galois field $F_{q}$ of size $q$. Let $\mathcal{P}_{j}=$ $\left\{i \mid c_{j, i}=1\right\}$ denote the indices of data packets that $y_{j}$ carries, and let $d_{j}=\left|\mathcal{P}_{j}\right|$ denote the cardinality of $\mathcal{P}_{j}$. The notation $d_{j}$ is referred as the degree of coded packet $y_{j}$, which is also the weight of its coding vector $\mathbf{c}_{j}$.

The selection of coding coefficients will determine the complexity of the encoding and decoding process as well as the successful decoding probability. The measures may include minimizing the degree of coded packets so as to increase the sparsity of coding vectors. To this end, SSNC uses a systematic network coding framework and can be separated into two phases. In the first phase, the BS encodes the packets in a systematic manner, which means the $j$-th SCP will only contain the information 
of the $j$-th data packet, i.e. $c_{j, i}=\mathbf{1}(j=i)$ and $\mathcal{P}_{j}=\{j\}, j=1, \cdots, k$. Therefore, these first $k$ coded packets can be termed as systematic SCPs with degree 1 . They are instantaneously decodable and help the receiver nodes to speed up the decoding process.

In the second phase, a number of $r$ random generated SCPs are produced, i.e., the coding coefficients of $\mathbf{c}_{j}, j=k+2, \cdots, n$ are randomly selected in $F_{q}$. In this article, we adopt the binary finite field $F_{2}=\{0,1\}$, and the coding coefficients should either be one or zero. To increase the sparsity of coding vectors, the coding coefficients can be assume to be independent and identically distributed with the following probability distribution [13]

$$
\mathbb{P}\left(c_{i, j}=t\right)= \begin{cases}p_{0}, & t=0 \\ \frac{1-p_{0}}{q-1}, & t \in F_{q} \backslash\{0\}\end{cases}
$$

where $p_{0} \in\left(q^{-1}, 1\right)$ is the probability that a coding coefficient is zero and called the sparsity of the coding vector. As $p_{0}$ increases, the expected degree of SCPs will decrease, which means the operation required for encoding will also decrease. However, larger $p_{0}$ will make the coding vectors of the randomly generated SCPs too sparse to contribute new data packets during the decoding process. Henceforth, $p_{0}$ may have its optimal value and should be designed accordingly.

An IIoT node will initiate the decoding process when it cannot receive more packets from its neighbors. In the receiving packet queue of the node, there may be systematic and random generated SCPs. The node will first decode all the received systematic SCPs into data packets. Next, it will try to decode the randomly generated SCPs with sparse coding vectors. An SCP is said to be available for decoding if its coding vector contains only one new non-zero coefficient with respect to the decoded data packets. The decoding process will be iteratively performed until there are no available SCPs to be decoded.

\subsubsection{Cooperative coded forwarding (CCF)}

The operation of cooperative coded forwarding is described as follows. An IIoT node will either forward or ignore a newly received SCP with probability $\omega \in(0,1)$ or $1-\omega$, respectively. After the nodes have received more than two SCPs, it will also begin the cooperative coded forwarding procedure. The node first chooses two different SCPs $y_{a}$ and $y_{b}$ from its received queue. It will directly encodes them into a relay coded packet $(\mathrm{RCP}) z_{a, b}=y_{a} \oplus y_{b}$ with probability $\xi \in(0,1)$ using the exclusive or (XOR) method. Since the SCPs are encoded in $G F(2)$, the XOR operation is equivalent to the addition process in SSNC. Even if a node have received neither $y_{a}$ nor $y_{b}$, the reception of $z_{a, b}$ can still be considered as an input packet of the decoding process of SSNC.

\section{Performance analysis model}

The SCPs generated by SSNC are processed and forwarded by the CCF mechanism. Therefore we first analyze the successful reception probability provided by CCF and then the successfully decoding probability of SSNC packets at the IIoT nodes. 


\subsection{Successful reception probability of CCF}

4.1.1 The percolation condition of probabilistic forwarding under probability $\omega$

The performance of probabilistic forwarding can be modeled by using the percolation theory [21]. We start our analysis of broadcasting from the simplest case of an ideal flooding scenario without channel outage. In this scenario, all the nodes are effective relay nodes. Denote the set of nodes that can connect to the origin $W_{\mathbf{0}}$ containing the origin $\mathbf{0}$. Let $\eta=\left|W_{\mathbf{0}}\right| / N$ denote the fraction of network nodes that are able to connect to the origin. where $\left|W_{\mathbf{0}}\right|$ is the cardinality of $W_{\mathbf{0}}$. The notation $\eta$ is also the probability that a network node can successfully receive a typical packet.

As $\varphi$ increases, $\eta$ also increase as there are more nodes in $W_{\mathbf{0}}$. Define $\eta(\varphi)$ as a function of $\varphi$. Kumar et al. [12] introduces the concept of "near-broadcast" when the value of $\eta$ is close to 1 . Given $\epsilon \in(0,1)$, let $\varphi_{\epsilon}$ denote the minimum value of $\varphi$ that a near-broadcast could happen, which is given as

$$
\varphi_{\epsilon}=\inf \{\varphi \mid \mathbb{E}[\eta(\varphi)]>1-\epsilon\}
$$

The goal of this subsection is to determine the value of $\varphi_{\epsilon}$ with given $N$ and $\epsilon$, so as to appropriately set the parameters of $\omega$ and $\xi$. However, it turns out that it is quite difficult to find because of the dominance of complicated boundary effects [22], and the closed-form expression of $\eta(\varphi)$ is not given in the literature. Thus we turn to the simulation results of $\eta(\varphi)$, which is provided in [23] by setting $N=100$, and finally approximate it by a Weibull distribution given as

$$
\eta(\varphi) \simeq 1-e^{-(2 \varphi / 3 \pi)^{\pi}}
$$

The simulation and approximated results of $\eta$ are shown in Fig. 3, which validates the accuracy of the approximation. We notice that the value of $\epsilon$ should be sufficiently small, as the value of $\varphi_{\epsilon}$ needs to be greater than the critical mean node degree $a_{c}$ for continuum percolation [20], which is crucial for effective broadcast transmission. The range of such critical mean node degree is provided in [20] as $4.508<a_{\mathrm{c}}<4.515$, and a more precise result is given in [23] as $a_{\mathrm{c}} \approx 4.512$.

Next we consider the case that only a fraction of nodes will forward the packets after successful reception under perfect channels, i.e. $\omega<1$ and $\rho=1$. The volunteer relay nodes provide coverage of the silent nodes in the rest of the network, which can be modeled as a site percolation [24]. To analyze the value of connected probability $\eta$ in such case, we first remove the silent nodes and calculate the connected probability of the volunteer relay nodes, which is denoted as $\eta_{\mathrm{r}}$. The value of $\eta_{\mathrm{r}}$ can be calculated by substitute the mean degree of volunteer relay nodes $\varphi_{\mathrm{r}}=\omega \varphi$ into Eq. (4). As a result, we have $\omega \geq \varphi_{\epsilon} / \lambda \pi \delta^{2}$. Then we randomly scatter the rest silent nodes back to the scenario. Let $\eta_{\mathrm{s}}$ denote the probability that a randomly placed silent node can be covered by a relay node in the connected component $W_{\mathbf{0}}$, which can be approximated as $\eta_{\mathrm{s}} \simeq \eta_{\mathrm{r}}$ when $\eta_{\mathrm{r}}$ is large enough. And the connected probability of the whole network should be

$$
\eta_{\omega}=\omega \eta_{\mathrm{r}}+(1-\omega) \eta_{\mathrm{s}} \simeq \eta_{\mathrm{r}}=\eta(\omega \varphi)
$$




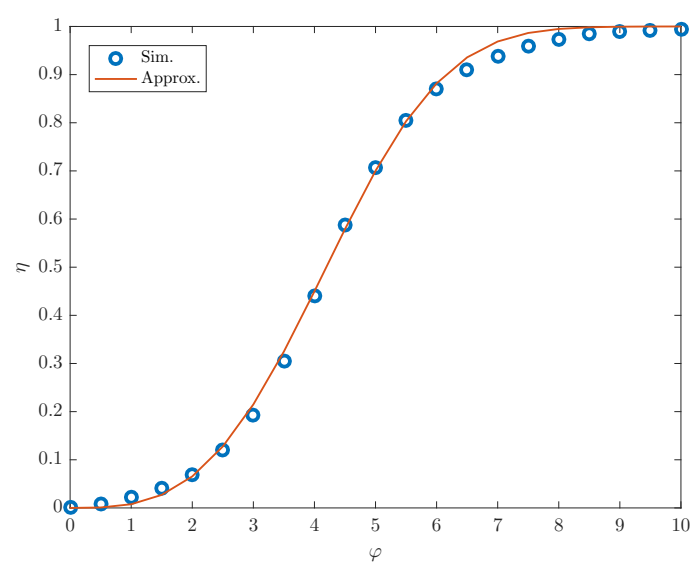

Figure 3 Simulation and approximated results of $\eta(\varphi), N=100$.

Finally, we turn to a practical case where wireless links between nodes are intermittent with $\rho>0$. This can be considered as a bond percolation [24] and is equivalent to a site percolation where the relay nodes proactively forward the SCPs with probability $\omega_{\rho}=\omega \rho$. Thus we can substitute the direct forward probability $\omega$ by $\omega_{\rho}$ in Eq. (5) to obtain the result of successful reception probability.

\subsubsection{The effect of coded forwarding under probability $\xi$}

Let $G_{j}=\left\langle V_{j}, E_{j}\right\rangle$ denote the connected forwarding graph of the $j$-th SCP $y_{j}$, where $V_{j}$ is the set of nodes that has received $y_{j}$, and $E_{j}$ contains the links that have successfully delivered $y_{j}$. Consider two different forwarding graph $G_{a}$ and $G_{b}$, and a common node $v \in V_{a} \cap V_{b}$. Denote the nodes covered by $v$ in the two graphs as $\mathcal{N}_{a}(v)=\mathcal{N}(v) \cap V_{a}$ and $\mathcal{N}_{b}(v)=\mathcal{N}(v) \cap V_{b}$ respectively. Suppose $v$ is going to encode $y_{a}$ and $y_{b}$ into a RCP $z_{a, b}=y_{a} \oplus y_{b}$ and forward it to its one-hop neighbors. A one-hop neighbor node $u \in \mathcal{N}(v)$ of $v$ can receive $z_{a, b}$ with probability $1-\rho$ due to the packet erasure effect. If $u$ has already received $y_{a}$ and $y_{b}$, i.e. $u \in \mathcal{N}_{a}(v) \cap \mathcal{N}_{b}(v)$, the receiving of $z_{a, b}$ become redundant. If $u$ has only received $y_{a}$, the receiving of $z_{a, b}$ helps it to decode $y_{b}$, and the node $u$ is added to $V_{b}$. The link $e(u, v)$ will also be involved in $E_{b}$. Similarly, the receiving of $y_{b}$ and $z_{a, b}$ will add $u$ into $V_{a}$. If $u$ have received neither $y_{a}$ nor $y_{b}$, the receiving of $z_{a, b}$ will not change $G_{a}$ or $G_{b}$. The effect of this coded forwarding action on the broadcast performance can therefore be regarded as the modification of the two corresponding forwarding graphs $G_{a}$ and $G_{b}$, which is shown in Fig. 4 .

To quantify the modification effect on the forwarding graphs, we analyze the relationship between the coded forwarding probability $\xi$ and the number of decoded SCPs at an IIoT node. Let $r$ denote the number of decoded SCPs at a node. Let $c$ denote the number of received RCPs at a node. The decoding process of RCPs can be modeled as a Markov chain consisting of a series of state $s_{n}(r)$. Denote the single step transition probability from $s_{n}(r)$ to $s_{n}(r+i)$ as $p_{n}(r, r+i)$, whose 


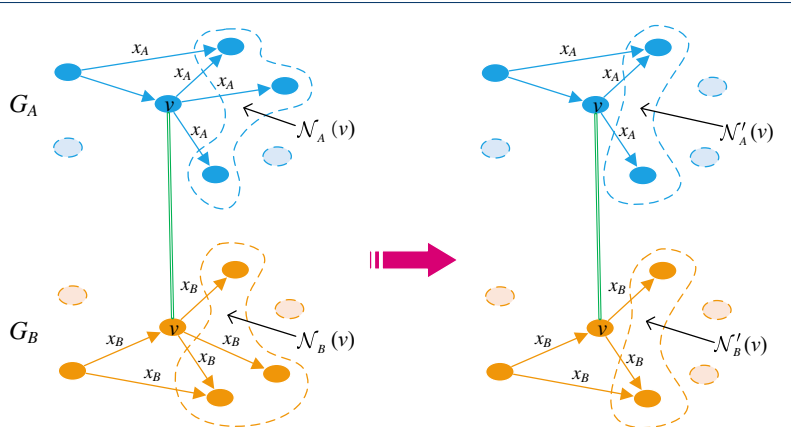

Figure 4 The modification effect of cooperative coded forwarding on percolation graphs.

expression is given as

$$
p_{n}(r, r+i)= \begin{cases}\frac{\left(\begin{array}{c}
r \\
1
\end{array}\right)\left(\begin{array}{c}
n-r \\
1
\end{array}\right)}{\left(\begin{array}{c}
n \\
2
\end{array}\right)}=\frac{2 r(n-r)}{n(n-1)}, & i=1, r=1,2, \cdots, n-1 \\
1-\frac{2 r(n-r)}{n(n-1)}, & i=0, r=1,2, \cdots, n \\
0, & \text { otherwise }\end{cases}
$$

Let $\mathbf{P}_{n \times n}=\left\{p_{n}(i, j) \mid i, j=1,2, \cdots, n\right\}$ denote the transition probability matrix. Let $\mathbf{w}^{(i)}=\left\{w^{(i)}(n, r) \mid r=0,1, \cdots, n\right\}$ denote the probability distribution of $r$ after $i$ steps. Since $r$ is a binomial random variable of parameter $n$ and $\eta$, i.e. $r \sim \operatorname{Bin}(n, \eta)$, the probability distribution of $r$ before decoding RCPs can be written as

$$
w^{(0)}(n, r)=\left(\begin{array}{l}
n \\
r
\end{array}\right) \eta^{r}(1-\eta)^{n-r}
$$

After decoding a number of $c$ RCPs, the probability distribution of $r$ is written as

$$
\mathbf{w}^{(c)}=\mathbf{w}^{(0)} \cdot \mathbf{P}_{n \times n}^{c}
$$

Therefore we obtain the expectation of $r$, which is given as

$$
\bar{r}(n, c) \triangleq \mathbb{E}[r(n, c)]=\sum_{r=0}^{n} w^{(c)}(n, r) \cdot r
$$

The number of RCPs generated at a node is related to the number of decoded SCPs $r$ and the coded forwarding probability $\xi$. Whenever the nodes have decoded new SCPs from the received RCPs, it will continue to produce some new RCPs afterward. This iterative process can be asymptotically analyzed and finally approaches the relationship between $\xi$ and the mean number of decoded SCPs. Define the mean number of RCPs generated at a node that has received $r$ out of $n$ SCPs as $C(r, \xi)=\left(\begin{array}{l}r \\ 2\end{array}\right) \cdot \xi=\frac{\xi \cdot r \cdot(r-1)}{2}$. Let $r_{i}$ denote the number of decoded SCPs after the $i$-th iteration, and let $c_{i}$ denote the number of RCPs participated in the decoding process in the $i$-th iteration. Clearly,we have $r_{0}=r$, and the expression of $c_{i}$ is 
written as

$$
c_{i}= \begin{cases}C\left(r_{i-1}, \xi\right) & , i=1 \\ C\left(r_{i-1}, \xi\right)-C\left(r_{i-2}, \xi\right) & , i>1\end{cases}
$$

When $c_{i}<1$, i.e. $\left(r_{i-1}-1\right) r_{i-1}-\left(r_{i-2}-1\right) r_{i-2}<2 \xi^{-1}$, the value of $r_{i}$ may not increase and so the iteration terminates.

\subsection{Successfully decoding probability of SSNC packets}

Consider that each IIoT node receives an arbitrary SCP with the same successful reception probability $\eta_{\xi}$. Denote the number of received systematic and random SCPs at a typical IIoT node as $R_{s}$ and $R_{r}$, respectively. $R_{s}$ is binomial random variable with parameters $k$ and $\eta_{\xi}$, i.e. $R_{s} \sim \operatorname{Bin}\left(k, \eta_{\xi}\right)$. Similarly, we have $R_{r} \sim$ $\operatorname{Bin}\left(n-k, \eta_{\xi}\right)$.

Let $\tau$ denote the number of decoded data packets from the random generated SCPs, thus the expectation of successful decoding probability $p$ can be written as

$$
\mathbb{E}[p]=\mathbb{E}\left[\frac{R_{s}+\tau}{k}\right]=\mathbb{E}\left[\frac{R_{s}}{k}\right]+E\left[\frac{\tau}{k}\right]=\eta_{\xi}+\frac{\mathbb{E}[\tau]}{k}
$$

where the expectation of $\tau$ is derived as follows.

Suppose that an IIoT node has decoded $m<k$ data packets, which forms the collection of decoded data packets $\mathcal{D}$ of size $|\mathcal{D}|=m$. Consider a randomly generated SCP of degree $d \in[1, m]$, it can be successfully decoded by this IIoT node only when one out of $d$ data packets it carried have not been decoded. If all the data packets carried by this SCP have been already decoded, this SCP becomes redundant and should be ignored in the future decoding process. The probability of these two conditions are written as

$$
\begin{aligned}
& p_{\text {dec }}(m, d)=\frac{\left(\begin{array}{c}
m \\
d-1
\end{array}\right)\left(\begin{array}{c}
k-m \\
1
\end{array}\right)}{\left(\begin{array}{l}
k \\
d
\end{array}\right)}=\frac{m !(k-d) !(k-m) d}{(m-d+1) ! k !} \\
& p_{\text {ign }}(m, d)=\frac{\left(\begin{array}{c}
m \\
d
\end{array}\right)}{\left(\begin{array}{l}
k \\
d
\end{array}\right)}=\frac{m !(k-d) !}{k !(m-d) !}
\end{aligned}
$$

Since the degree of a random generated SCP $d$ is a binomial function of parameter $k$ and $1-p_{0}$, the expected successful decoding probability and ignored probability of an arbitrary random SCP are written as

$$
\begin{aligned}
p_{\text {dec }}(m) & =\sum_{d=1}^{m} \mathbb{P}\left(d_{j}=d\right) \cdot p_{\text {dec }}(m, d) \\
& =\sum_{d=1}^{m} \frac{m !(k-m)}{(d-1) !(m-d+1) !} p_{0}^{k-d}\left(1-p_{0}\right)^{d}
\end{aligned}
$$




$$
\begin{aligned}
p_{\text {ign }}(m) & =\sum_{d=1}^{m}\left(d_{j}=d\right) \cdot p_{\text {ign }}(m, d) \\
& =\sum_{d=1}^{m} \frac{m !}{d !(m-d) !} p_{0}^{k-d}\left(1-p_{0}\right)^{d}
\end{aligned}
$$

Let $S_{m_{\mathrm{s}}, m_{\mathrm{r}}}$ denote the decoding state of an IIoT node, where $m_{\mathrm{s}}$ is the number of decoded data packets and $m_{\mathrm{r}}$ is the number of random generated SCPs that are not processed. An IIoT node at state $S_{m_{\mathrm{s}}, m_{\mathrm{r}}}$ will try to decode the unprocessed random SCPs when $m_{\mathrm{s}}<k$ and $m_{\mathrm{r}} \geq 1$. If there is at least one decodable SCP, the node will switch to state $S_{m_{\mathrm{s}}+1, m_{\mathrm{r}}-1}$. Otherwise, the node will stay at the current state. Regarding the ignored packets, the transition probabilities can be written as

$$
\begin{gathered}
P\left(S_{m_{\mathrm{s}}+1, m_{\mathrm{r}}-1} \mid S_{m_{\mathrm{s}}, m_{\mathrm{r}}}\right) \triangleq 1-\sum_{m_{\mathrm{i}}=0}^{m_{\mathrm{r}}} \mathbb{P}\left(m_{\mathrm{i}}=m \mid S_{m_{\mathrm{s}}, m_{\mathrm{r}}}\right) \cdot\left(1-p_{\mathrm{dec}}\left(m_{\mathrm{s}}\right)\right)^{m_{\mathrm{r}}-m} \\
P\left(S_{m_{\mathrm{s}}, m_{\mathrm{r}}} \mid S_{m_{\mathrm{s}}, m_{\mathrm{r}}}\right) \triangleq 1-P\left(S_{m_{\mathrm{s}}+1, m_{\mathrm{r}}-1} \mid S_{m_{\mathrm{s}}, m_{\mathrm{r}}}\right)
\end{gathered}
$$

where $\mathbb{P}\left(m_{\mathrm{i}}=m \mid S_{m_{\mathrm{s}}, m_{\mathrm{r}}}\right)=\left(\begin{array}{c}m_{\mathrm{r}} \\ m_{\mathrm{i}}\end{array}\right) p_{\text {ign }}^{m_{\mathrm{i}}}\left(m_{\mathrm{s}}\right)\left(1-p_{\text {ign }}\left(m_{\mathrm{s}}\right)\right)^{m_{\mathrm{r}}-m_{\mathrm{i}}}$, number $m_{i}$ is a binomial stochastic variable of parameter $m_{\mathrm{r}}$ and $p_{\text {ign }}\left(m_{\mathrm{S}}\right)$.

As $R_{s}$ and $R_{r}$ are two independent binomial random variables, an IIoT node will start its decoding process at state $S\left(R_{\mathrm{s}}, R_{\mathrm{r}}\right)$ with probability

$$
P_{\text {start }}\left(S_{R_{\mathrm{s}}, R_{\mathrm{r}}}\right)=\mathbb{P}\left[m_{\mathrm{s}}=R_{\mathrm{s}}\right] \cdot \mathbb{P}\left[m_{\mathrm{r}}=R_{\mathrm{r}}\right]=\left(\begin{array}{c}
k \\
R_{\mathrm{s}}
\end{array}\right)\left(\begin{array}{c}
n-k \\
R_{\mathrm{r}}
\end{array}\right) \eta_{\xi}^{R_{\mathrm{s}}+R_{\mathrm{r}}}\left(1-\eta_{\xi}\right)^{n-R_{\mathrm{s}}-R_{\mathrm{r}}}
$$

and continue decoding until either all the $R_{\mathrm{r}}$ random SCPs have been processed or the all the $k$ data packets have been decoded. Thus the maximum number of $\tau$ should be $\tau_{\max }=\min \left(k-R_{\mathrm{s}}, R_{\mathrm{r}}\right)$. The steady state probability of $\tau$-th state $S\left(R_{\mathrm{S}}+\tau, R_{\mathrm{r}}-\tau\right)$ can be written as

$$
P\left(S_{R_{\mathrm{s}}+\tau, R_{\mathrm{r}}+\tau}\right)= \begin{cases}P\left(S_{R_{\mathrm{s}}+\tau, R_{\mathrm{r}}+\tau} \mid S_{R_{\mathrm{s}}+\tau, R_{\mathrm{r}}+\tau}\right) & , \tau=0 \\ \left(\prod_{t=0}^{\tau-1} P\left(S_{R_{\mathrm{s}}+t+1, R_{\mathrm{r}}+t+1} \mid S_{R_{\mathrm{s}}+t, R_{\mathrm{r}}+t}\right)\right) & , \tau \in\left[1, \tau_{\max }\right] \\ \cdot P\left(S_{R_{\mathrm{s}}+\tau, R_{\mathrm{r}}+\tau} \mid S_{R_{\mathrm{s}}+\tau, R_{\mathrm{r}}+\tau}\right) & \end{cases}
$$

Finally, the expectation of $\tau$ can be written as

$$
\mathbb{E}[\tau]=\sum_{R_{\mathrm{s}}=0}^{k} \sum_{R_{\mathrm{r}}=1}^{n-k} P_{\text {start }}\left(S_{R_{\mathrm{s}}, R_{\mathrm{r}}}\right) \cdot \sum_{\tau=0}^{\tau_{\max }} \tau \cdot P\left(S_{R_{\mathrm{s}}+\tau, R_{\mathrm{r}}+\tau}\right)
$$

Substitute Eq. (20) into Eq. (11) we can have the mean successful decode probability at an IIoT node of a data packet generation of size $k$.

\subsection{Total transmission number}

According to the design of PCCF, the total transmission number of a data packet generation is given as

$$
T=N \cdot\left(n \eta \cdot \omega+\frac{n \eta_{\xi}\left(n \eta_{\xi}-1\right)}{2} \cdot \xi\right)
$$




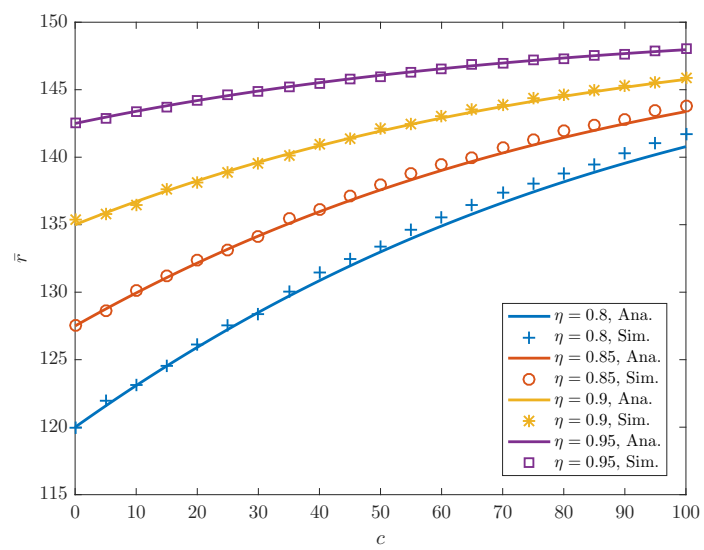

Figure 5 Simulation and analytical results of the relationships between $c$ and $\bar{r}(n, c)$.

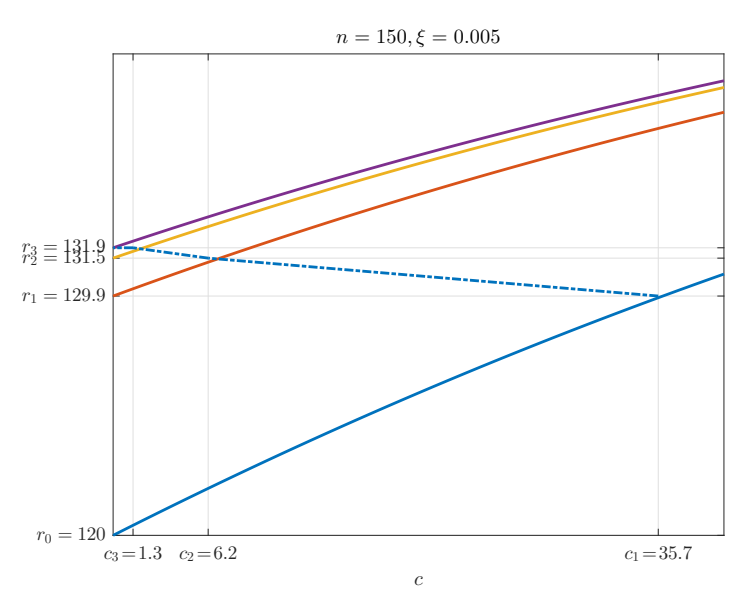

Figure 6 Demonstration of the asymptotic analysis of $r_{i}$ and $c_{i}$.

which will increase as $\eta$ and $\xi$ increases.

\section{Results and discussion}

\subsection{Successful reception probability of CCF}

5.1.1 The effect of coded forwarding under probability $\xi$

We compare the simulation and analytical results of the relationships between $c$ and $\bar{r}(n, c)$ under $n=150$ and $\eta \in\{0.8,0.85,0.9,0.95\}$ in Fig. 5.

As the value of $\bar{r}(n, c)$ may not be an integer, we denote $r_{i}=\left\lceil\bar{r}\left(n, c_{i-1}\right)\right\rceil$ for the simplicity of notation, where $\lceil\cdot\rceil$ is the ceiling function. When $c_{i}<1$, i.e. $\left(r_{i-1}-1\right) r_{i-1}-\left(r_{i-2}-1\right) r_{i-2}<2 \xi^{-1}$, the value of $r_{i}$ may not increase and so the iteration terminates. An demonstration of the numerical analysis is presented in Fig. 6.

We can quantify the modification effect on the forwarding graphs based on the relationships between $\xi$ and the successful reception probability of SCPs $\eta_{\xi}=\bar{r} / n$ at a IIoT node, which is shown in Fig. 7. We observe that the larger the value of $n$ 


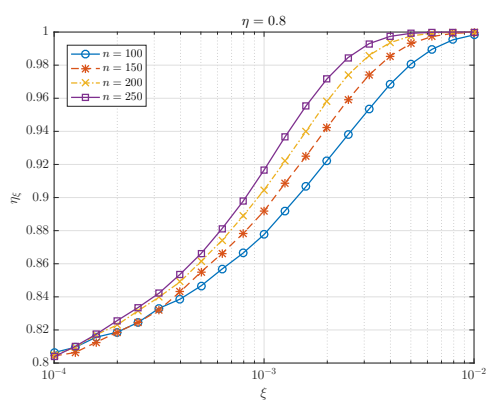

(a)

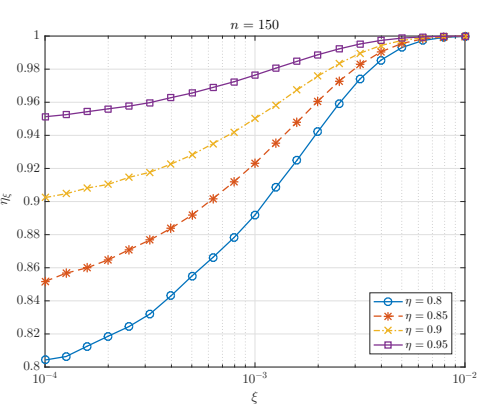

(b)

Figure 7 Relationship between $\xi$ and $\eta_{\xi}$.

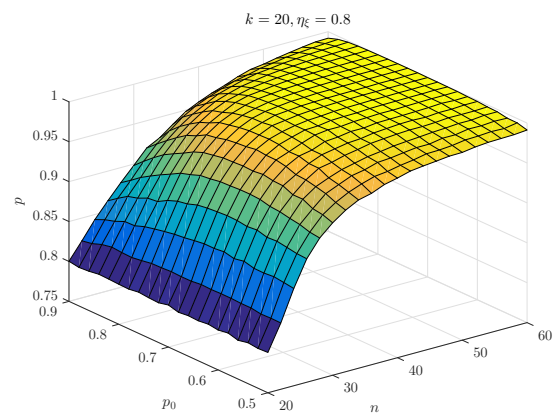

(a) $k=20$

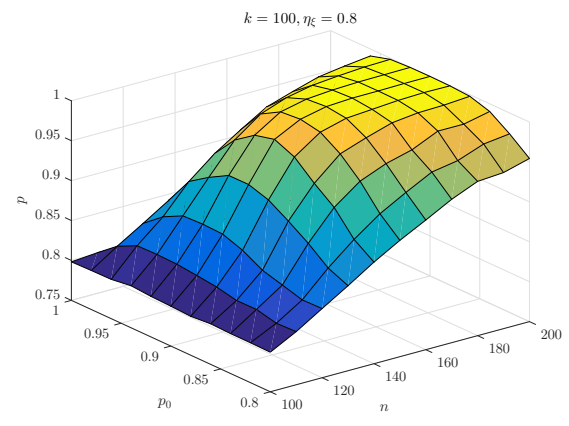

(b) $k=100$

Figure 8 Simulation results of successful decoding probability $p$ under $\eta_{\xi}=0.8$.

is, the faster $\eta_{\xi}$ increases as $\xi$ grows. Also the contribution of the increasing of $\xi$ is much higher with a smaller $\eta$.

\subsection{Successfully decoding probability of SSNC packets}

The simulation results of successful decoding probability $p$ are shown in Fig. 8, where we can observe that the optimal sparsity $p_{0, \text { opt }}$ for SSNC is approximately 0.7 when $k=20$, and 0.9 when $k=100$. This means that the optimal sparsity should be increased as the size of data packet generation increases.

Fig. 9 demonstrates the analytical and simulation results of $p$, which validates the accuracy of the analytical model in Sect. 4.

\subsection{Total transmission number}

The results of the successful reception probability $\eta_{\xi}$ and the transmission number $T$ are given in Fig. 10(a) and Fig. 10(b) respectively. We can see that in order to achieve the same value of $\eta_{\xi}$, there exists a smallest transmission number and the corresponding optimal values of $\omega$ and $\xi$ to fulfill the near-broadcast requirement of mobile edge networks. 


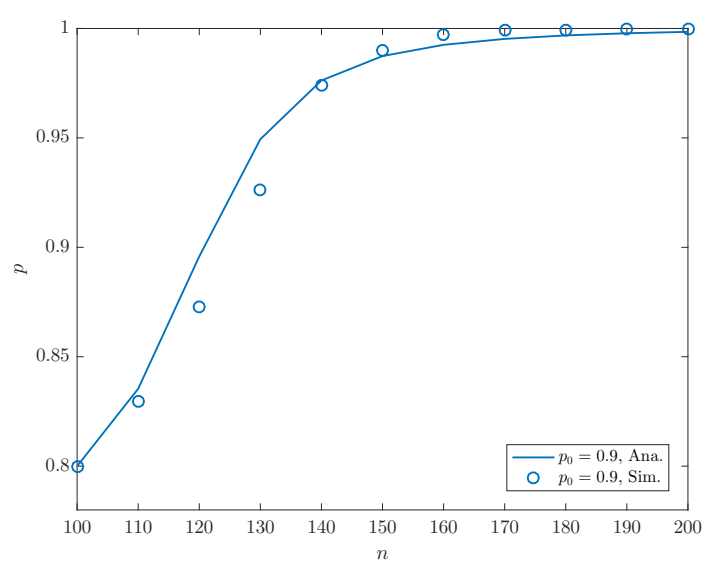

Figure 9 Comparison of analytical and simulation results of successful decoding probability.

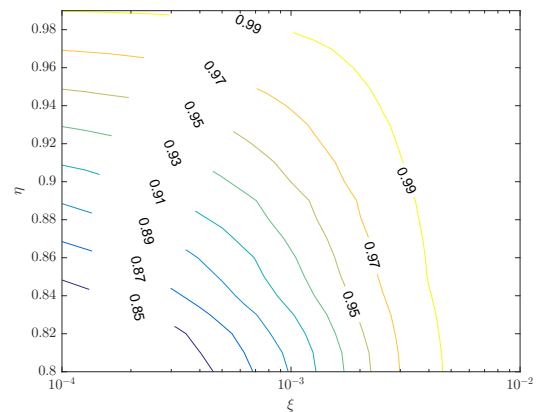

(a) $\eta_{\xi}$

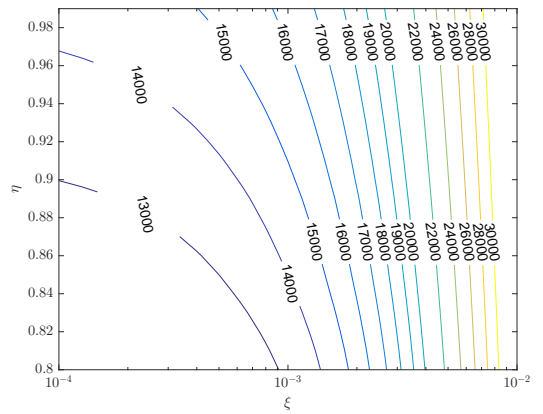

(b) $T$

Figure 10 Comparison of the contour maps of $\eta_{\xi}$ and $T$ under $n=150$.

\section{Conclusion}

In this article, the network coding technique is adopted for realizing efficient broadcasting in MEC-enabled IIoT networks. To minimize the transmission number at the data source, the systematic network coding framework is used and the sparsity of random generated coded packets is optimized. To increase the efficiency of re-encoding process at the relay nodes, we analyze the impact of direct and coded forwarding probability on the reception performance at the network nodes, which gives fundamental results for further optimization. Future researches include the design of cooperative coded transmission among multiple data sources and the performance analysis under hierarchical network settings.

\section{Appendix}

Abbreviations

MEC: Mobile edge computing; 5G: Fifth generation; IloT: Industrial internet of things; UAV: Unmanned aerial vehicle; BS: Base station; D2D: Device-to-device; NC: Network coding; RLNC: Random linear network coding; GF: Galois field; PCCF: Probabilistic cooperative coded forwarding; SSNC: Systematic sparse network coding; CCF: Cooperative coded forwarding; SCP: Source coded packet; RCP: Relay coded packet. 
Acknowledgements

The authors gratefully acknowledge the valuable comments and suggestions of the reviewers, which have improved the presentation of this article.

Authors' contribution

The authors have contributed jointly to the manuscript. All authors have read and approved the final manuscript.

Funding

This work is supported by the Fundamental Research Funds for the Central Universities under Grant 2021JBZ102.

Availability of data and materials

Data sharing not applicable to this article during the current study.

Competing interests

The authors declare that they have no competing interests.

Author details

School of Electronic and Information Engineering, Beijing Jiaotong University, Beijing, P. R. China.

\section{References}

1. Ma, Z., Xiao, M., Xiao, Y., Pang, Z., Poor, H.V., Vucetic, B.: High-Reliability and Low-Latency Wireless Communication for Internet of Things: Challenges, Fundamentals, and Enabling Technologies. IEEE Internet Things J. 6(5), 7946-7970 (2019). doi:10.1109/JIOT.2019.2907245

2. Corcoran, P., Datta, S.K.: Mobile-Edge Computing and the Internet of Things for Consumers: Extending cloud computing and services to the edge of the network. IEEE Consumer Electron. Mag. 5(4), 73-74 (2016). doi:10.1109/MCE.2016.2590099

3. Liu, Y., Peng, M., Shou, G., Chen, Y., Chen, S.: Toward Edge Intelligence: Multiaccess Edge Computing for 5G and Internet of Things. IEEE Internet Things J. 7(8), 6722-6747 (2020). doi:10.1109/JIOT.2020.3004500

4. Li, L., Ramjee, R., Buddhikot, M., Miller, S.: Network Coding-Based Broadcast in Mobile Ad-hoc Networks. In: IEEE INFOCOM 2007 - 26th IEEE International Conference on Computer Communications, pp. 1739-1747. IEEE, Anchorage, AK, USA (2007). doi:10.1109/INFCOM.2007.203

5. Song, H., Liu, L., Pudlewski, S.M., Bentley, E.S.: Random Network Coding Enabled Routing Protocol in Unmanned Aerial Vehicle Networks. IEEE Trans. Wireless Commun. 19(12), 8382-8395 (2020). doi:10.1109/TWC.2020.3022399

6. Song, H., Liu, L., Shang, B., Pudlewski, S., Bentley, E.S.: Enhanced Flooding-Based Routing Protocol for Swarm UAV Networks: Random Network Coding Meets Clustering. In: IEEE INFOCOM 2021 - IEEE Conference on Computer Communications, pp. 1-10. IEEE, Vancouver, BC, Canada (2021). doi:10.1109/INFOCOM42981.2021.9488721

7. Shi, L., Cai, K., Mei, Z.: Linear Network Coded Computation in Mobile Edge Computing. In: 2019 IEEE Global Communications Conference (GLOBECOM), pp. 1-6. IEEE, Waikoloa, HI, USA (2019). doi:10.1109/GLOBECOM38437.2019.9014018

8. He, W., Su, Y., Xu, X., Luo, Z., Huang, L., Du, X.: Cooperative Content Caching for Mobile Edge Computing With Network Coding. IEEE Access 7, 67695-67707 (2019). doi:10.1109/ACCESS.2019.2917977

9. Wang, L., Liu, Y., Xu, J., Yin, J., Xu, L., Yang, Y.: Network coding for reliable video distribution in multi-hop device-to-device communications. J Wireless Com Network 2020(1), 253 (2020). doi:10.1186/s13638-020-01869-0

10. Zhan, C., Gao, K.: Conflict-Free Scheduling for Partially Connected D2D Networks With Network Coding. IEEE Wireless Commun. Lett. 5(5), 456-459 (2016). doi:10.1109/LWC.2016.2585487

11. Papanikos, N., Papapetrou, E.: Deterministic Broadcasting and Random Linear Network Coding in Mobile Ad Hoc Networks. IEEE/ACM Trans. Networking 25(3), 1540-1554 (2017). doi:10.1109/TNET.2016.2641680

12. Kumar, B.R.V., Kashyap, N.: Probabilistic Forwarding of Coded Packets on Networks. IEEE/ACM Trans. Networking, 1-14 (2020). doi:10.1109/TNET.2020.3031467

13. Chen, W., Lu, F., Dong, Y.: The Rank Distribution of Sparse Random Linear Network Coding. IEEE Access 7 43806-43819 (2019). doi:10.1109/ACCESS.2019.2907005

14. Katti, S., Rahul, H., Wenjun Hu, Katabi, D., Medard, M., Crowcroft, J.: XORs in the Air: Practical Wireless Network Coding. IEEE/ACM Trans. Networking 16(3), 497-510 (2008). doi:10.1109/TNET.2008.923722

15. Yue, G., Yang, K., Zhao, S., Poor, H.V.: Design of Network Coding for Wireless Broadcast and Multicast With Optimal Decoders. IEEE Trans. Wireless Commun. 17(10), 6944-6957 (2018) doi:10.1109/TWC.2018.2864996

16. Sehat, H., Pahlevani, P.: An Analytical Model for Rank Distribution in Sparse Network Coding. IEEE Commun. Lett. 23(4), 556-559 (2019). doi:10.1109/LCOMM.2019.2896626

17. Chen, W., Lu, F., Dong, Y.: Improved Expression for Rank Distribution of Sparse Random Linear Network Coding. IEEE Commun. Lett. 25(5), 1472-1476 (2021). doi:10.1109/LCOMM.2020.3041845

18. Shrader, B., Jones, N.M.: Systematic wireless network coding. In: MILCOM 2009 - 2009 IEEE Military Communications Conference, pp. 1-7. IEEE, Boston, MA, USA (2009). doi:10.1109/MILCOM.2009.5380081

19. Jones, A.L., Chatzigeorgiou, I., Tassi, A.: Binary systematic network coding for progressive packet decoding. In: 2015 IEEE International Conference on Communications (ICC), pp. 4499-4504. IEEE, London (2015). doi:10.1109/ICC.2015.7249031

20. Haenggi, M.: Stochastic Geometry for Wireless Networks. Cambridge University Press, ??? (2012)

21. Gang, W., XiaoRong, Z., HaiTao, C., LiZhi, L.: Research on the probabilistic broadcasting algorithms of Mobile ad hoc network based on percolation theory. In: 2011 International Conference on Mechatronic Science, Electric Engineering and Computer (MEC), pp. 330-333. IEEE, Jilin, China (2011). doi:10.1109/MEC.2011.6025468 
22. Penrose, M.D.: Onk-connectivity for a geometric random graph. Random Struct. Alg. 15(2), $145-164$ (1999). doi:10.1002/(SICI)1098-2418(199909)15:2¡145::AID-RSA2¿3.0.CO;2-G

23. Gu, X., Feng, H.: Connectivity analysis for a wireless sensor network based on percolation theory. In: 2010 International Conference on Computer Application and System Modeling (ICCASM 2010), vol. 5. IEEE, Taiyuan, China (2010). doi:10.1109/ICCASM.2010.5619163

24. Raman, V., Gupta, I.: Performance Tradeoffs Among Percolation-Based Broadcast Protocols in Wireless Sensor Networks. In: 2009 29th IEEE International Conference on Distributed Computing Systems Workshops, pp. 158-165. IEEE, Montreal, Quebec, Canada (2009). doi:10.1109/ICDCSW.2009.76

Figure Title and Legend

Figure. 1: Architecture of MEC-enabled industrial communication network

The figure displays network model. We consider a MEC-enabled IloT consisting of a base station (BS) equipped with a MEC server and $N$ randomly distributed IloT nodes in the edge network. The MEC server has direct connections with the core cloud with data centers. The lloT nodes in the edge network are assumed to be located inside a square $[0, B]^{2}$ on the ground where $B$ is the edge of the square, and the BS is located at the origin of the edge network square.

Figure. 2: Diagram of the network-coding based mechanisms of PCCF

The figure displays the scheme of systematic sparse network coding (SSNC) mechanism and cooperative coded forwarding(CCF). The probabilistic cooperative coded forwarding scheme consists of two network-coding based mechanisms. The BS deploys the systematic sparse network coding (SSNC) mechanism, in which it will organize $k$ data packets as a packet generation $\mathbf{x}=\left\{x_{i} \mid i=1, \cdots, k\right\}$ and encoded them into $n \geq k$ source coded packets (SCPs). It will broadcast the SCPs to the IloT nodes within its coverage. An IloT node which has receive the SCPs will perform cooperative coded forwarding (CCF), where it first directly forward the SCPs in a probabilistic manner, and then encode the received SCPs into some relay coded packets (RCPs) and broadcast them to its one-hop neighbors. In general, SSNC is a source-based network coding mechanism and CCF is related to the relay nodes.

Figure. 3: Simulation and approximated results of $\eta(\varphi), N=100$

The figure shows the simulation and approximated results of $\eta(\varphi), N=100$ and validates the accuracy of the approximation.

Figure. 4: The modification effect of cooperative coded forwarding on percolation graphs Let $G_{j}=\left\langle V_{j}, E_{j}\right\rangle$ denote the connected forwarding graph of the $j$-th SCP $y_{j}$, where $V_{j}$ is the set of nodes that has received $y_{j}$, and $E_{j}$ contains the links that have successfully delivered $y_{j}$. Consider two different forwarding graph $G_{a}$ and $G_{b}$, and a common node $v \in V_{a} \cap V_{b}$. Denote the nodes covered by $v$ in the two graphs as $\mathcal{N}_{a}(v)=\mathcal{N}(v) \cap V_{a}$ and $\mathcal{N}_{b}(v)=\mathcal{N}(v) \cap V_{b}$ respectively. Suppose $v$ is going to encode $y_{a}$ and $y_{b}$ into a RCP $z_{a, b}=y_{a} \oplus y_{b}$ and forward it to its one-hop neighbors. A one-hop neighbor node $u \in \mathcal{N}(v)$ of $v$ can receive $z_{a, b}$ with probability $1-\rho$ due to the packet erasure effect. If $u$ has already received $y_{a}$ and $y_{b}$, i.e.

$u \in \mathcal{N}_{a}(v) \cap \mathcal{N}_{b}(v)$, the receiving of $z_{a, b}$ become redundant. If $u$ has only received $y_{a}$, the receiving of $z_{a, b}$ helps it to decode $y_{b}$, and the node $u$ is added to $V_{b}$. The link $e(u, v)$ will also be involved in $E_{b}$. Similarly, the receiving of $y_{b}$ and $z_{a, b}$ will add $u$ into $V_{a}$. If $u$ have received neither $y_{a}$ nor $y_{b}$, the receiving of $z_{a, b}$ will not change $G_{a}$ or $G_{b}$. The effect of this coded forwarding action on the broadcast performance can therefore be regarded as the modification of the two corresponding forwarding graph $G_{a}$ and $G_{b}$

Figure. 5: Simulation and analytical results of the relationships between $c$ and $\bar{r}(n, c)$

The figure shows simulation and analytical results of the relationships between $c$ and $\bar{r}(n, c)$ under $n=150$ and $\eta \in\{0.8,0.85,0.9,0.95\}$.

Figure. 6: Demonstration of the asymptotic analysis of $r_{i}$ and $c_{i}$

The figure presents demonstration of the asymptotic analysis of $r_{i}$ and $c_{i}$ under $n=150, \eta=0.8$ and $\xi=0.005$.

Figure. 7: Relationship between $\xi$ and $\eta_{\xi}$

The figure presents the relationship between $\xi$ and $\eta_{\xi}:$ (a) $\eta=0.8$ and $n \in\{100,150,200,250\} ;$ (b) $n=150$ and $\eta \in\{0.8,0.85,0.9,0.95\}$. We can quantify the modification effect on the forwarding graphs through the relationships between $\xi$ and the successful reception probability of SCPs $\eta_{\xi}=\bar{r} / n$ at a IloT node. We observe that the larger the value of $n$ is, the faster $\eta_{\xi}$ increases as $\xi$ grows. Also the contribution of the increasing of $\xi$ is much higher with a smaller $\eta$.

Figure. 8: Simulation results of successful decoding probability $p$ under $\eta_{\xi}=0.8$

The figure presents simulation results of successful decoding probability $p$ under $\eta_{\xi}=0.8$. We can observe that the optimal sparsity $p_{0, \mathrm{opt}}$ for SSNC is approximately 0.7 when $k=20$, and 0.9 when $k=100$. This means that the optimal sparsity should be increased as the size of data packet generation increases.

Figure. 9: Comparison of analytical and simulation results of successful decoding probability

The figure shows comparison of analytical and simulation results of successful decoding probability under $k=100$, $\eta_{\xi}=0.8$ and $p_{0}=p_{0, \mathrm{opt}}=0.9$. It demonstrates the analytical and simulation results of $p$, which validates the accuracy of analytical model.

Figure. 10: Comparison of the contour maps of $\eta_{\xi}$ and $T$ under $n=150$

The figure shows comparison of the contour maps of $\eta_{\xi}$ and $T$ under $n=150$. We can see that in order to achieve the same value of $\eta_{\xi}$ with different transmission number, which indicates the existence of the smallest transmission number and the corresponding optimal values of $\omega$ and $\xi$ to fulfill the near-broadcast requirement of mobile edge networks. 